\title{
Two Unified Algorithms for Fundamental Planetary Ephemeris
}

\author{
Mohamed Adel Sharaf1, Abdel-Naby Saad Saad2,3, Aisha Abdu Alshaery4 \\ ${ }^{1}$ Department of Astronomy, Faculty of Science, King Abdulaziz University, Jeddah, KSA \\ ${ }^{2}$ Department of Astronomy, National Research Institute of Astronomy and Geophysics, Cairo, Egypt \\ ${ }^{3}$ Department of Mathematics, Preparatory Year, Qassim University, Buraidah, KSA \\ ${ }^{4}$ Department of Mathematics, College of Science for Girls, King Abdulaziz University, Jeddah, KSA \\ Email: Sharaf adel@hotmail.com, Saad6511@gmail.com, alshaary@hotmail.com
}

Received 28 September 2014; revised 25 October 2014; accepted 17 November 2014

Academic Editor: Michael Smith, University of Kent, UK

Copyright (C) 2014 by authors and Scientific Research Publishing Inc.

This work is licensed under the Creative Commons Attribution International License (CC BY).

http://creativecommons.org/licenses/by/4.0/

c) (i) Open Access

\begin{abstract}
In the present paper, we established two unified algorithms. The first algorithm is for the transformations between J2000.0 Keplerian orbital elements and B1950.0 elements, while the second is for the transformations between the equatorial orbital elements and the ecliptic orbital elements. Mathematica Modules of the algorithms are given together with some numerical applications.
\end{abstract}

\section{Keywords}

Spherical Astronomy, Precession, Ephemeris, Orbit Determination

\section{Introduction}

Precession is a change in the orientation of the rotational axis of a rotating body. In astronomy, precession refers to any of several slow changes in an astronomical body's rotational or orbital parameters, and especially to the Earth's precession of the equinoxes. The shift in the position of the Earth's axis and of the ecliptic caused by forces exerted by the Sun, Moon and planets not only causes a slight change in the angle between the equator and the ecliptic, but also a shift of the vernal equinox by about $1.5^{\circ}$ per century ( 1 ' per year). This effect is negligibly small for casual observing, but is an important correction for precise observations. To get accurate observations, the International Astronomical Union (IAU) 2000 recommended significant improvements in the definition of the International Celestial Reference System (ICRS) [1]. It follows that changes in Earth's orbital pa- 
rameters (e.g. orbital inclination, the angle between Earth's rotation axis and its plane of orbit) is important to the study of Earth's climate, in particular to the study of past ice ages.

For precise calculations therefore, the equinox of the coordinate system used must be stated. The equinoxes most frequently used are the equinox of date, equinox J2000 and equinox B1950 [2] and [3]. "Equinox of the date" means that the values used are those for the equator, ecliptic, and vernal equinox for actual date under consideration. Such daily alteration of the coordinate system is sensible if one requires the coordinates of a planet, for example, for use in conjunction with the setting circles on an equatorial mounted telescope, or on a transit circle. Since the shift in the Earth's axis, the orientation of the polar axis of a telescope will also alter. On the other hand, if one wants to study the actual spatial motion of a planet then it is better to use a fixed equinox, such as that for Julian Epoch J2000 (2000 January 1.5 = JD = 2451545.0), which was generally introduced in 1984. Before that, the older equinox B1950 has been used for a long time, and was employed for many stellar catalogues and atlases (such as the SAO Star Catalog and Atlas Coell). The beginning of the Basselian year 1950 (1950 Jan.0.9232 = JD 2433282.423).

On the other hand, there are two standard reference planes to specify the orbits of celestial objects, the equatorial plane of the Earth and the ecliptic plane (the plane of Earth's orbit around the Sun). However, due to precession, the equatorial and the ecliptic planes are slowly changing their positions relative to the background stars [4].

In this paper, we established two new unified computational algorithms each one is applicable to get ephemeris in both directions simultaneously. That is to mean it can be used as a switch between: 1) J2000.0 Keplerian orbital elements and B1950.0 elements and 2) between the equatorial orbital elements and the ecliptic orbital elements. Such artifices do not exist in any other numerical ephemeris methods. Furthermore, in our algorithms the number of the utilized equations was reduced via applying some mathematical operations, a matter, which facilitates the computations. Mathematica Modules for the two algorithms are also included.

Finally, it should mentioned that, although all numerical ephemeris methods utilize the same equations, but their accuracy may be differ greatly depending on a) the computational package adopted for their evaluation; b) the form of the equations, such that, the more they are explicit, the more their satiability and accuracy will be.

Due to the quite simple explicit forms of the reduced equations of our algorithms, and the usages of the most power full computational packages of Mathematica, consequently as regarding to that two points a \& b, our algorithms may be more accurate than that given by JPL system or other numerical ephemeris methods.

\section{Basic Formulations}

\subsection{Duality of Theorems Relating to the Spherical Triangle}

The duality of theorems relating to the spherical triangle [5] was stated as: Any theorem relating the sides and angles of any spherical triangle will remain true when the angles are changed into the supplements of the corresponding sides and the sides into the supplements of the corresponding angles.

\subsection{Transformation Formulae between J2000.0 and B1950.0 Keplerian Elements}

Given the six orbital elements $a, e, \tau, \omega, \Omega$, and $i$ in the B1950.0 reference system, one may compute the corresponding orbital elements for J2000.0-based system (dented by primes):

- $\quad a, a^{\prime}$ : Semi-major axes in B1950.0, J2000.0 systems.

- $e, e^{\prime}$ : Eccentricities in B1950.0, J2000.0 systems.

- $\tau, \tau^{\prime}$ : Pre-center passage times in B1950.0, J2000.0 systems.

- $\omega, \omega^{\prime}$ : Argument of pre-center in B1950.0, J2000.0 systems.

- $\Omega, \Omega^{\prime}$ : Longitude of the ascending node in B1950.0, J2000.0 systems.

- $\quad I, I^{\prime}$ : Inclination in B1950.0, J2000.0 systems.

where $0^{\circ} \leq \omega \leq 360^{\circ}, 0^{\circ} \leq \Omega \leq 360^{\circ}$ and $0^{\circ} \leq I \leq 180^{\circ}$ such that for direct motion $I$ ranges from $0^{\circ}$ to $90^{\circ}$; for retrograde motion ranges from $90^{\circ}$ to $180^{\circ}$. Clearly, $a=a^{\prime}, e=e^{\prime}, \tau=\tau^{\prime}$ while the angles $\omega^{\prime}, \Omega^{\prime}, I^{\prime}(\omega, \Omega, I)$ are computed from $\omega, \Omega, I\left(\omega^{\prime}, \Omega^{\prime}, I^{\prime}\right)$ as will be shown soon.

Figure 1 represents the relationship between B1950.0 and J2000.0 reference frames and the orbital plane. The numerical values for the angles $L^{\prime}, L, J$ [6] are $L^{\prime}=4^{\circ} .50001688, L=5^{\circ} .19856209$ and $J=0^{\circ} .00651966$. These values were computed using the values of $\varepsilon$ as $\varepsilon=23^{\circ} .44578787(\mathrm{~B} 1950.0)$ and $\varepsilon=23^{\circ} .43929111(\mathrm{~J} 2000.0)$. 


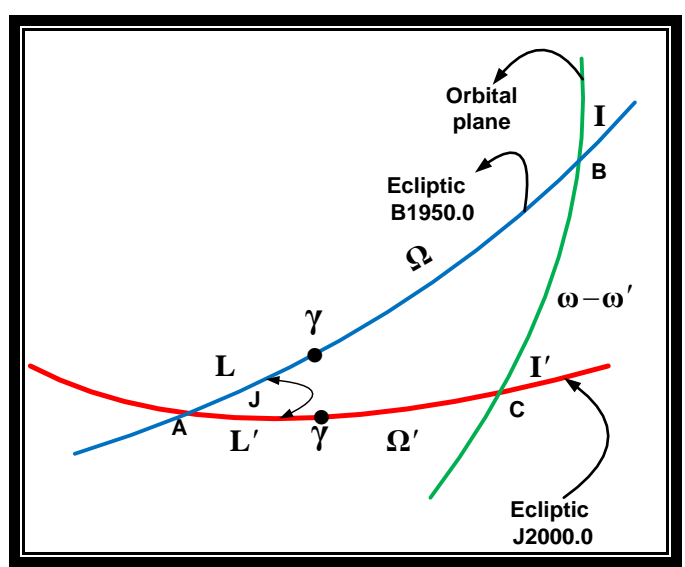

Figure 1. The relation between the reference systems B1950.0, J2000.0 and the orbital plane.

\subsubsection{The Basic Equations for the Transformation}

Applying the duality property (see Section 2.1) to the spherical triangle ABC of Figure 1, we get

$$
\begin{aligned}
& L+\Omega \stackrel{\text { to }}{\longrightarrow} 180-\left(180-I^{\prime}\right)=I^{\prime}, \\
& I \stackrel{\text { to }}{\longrightarrow} 180-\left(L^{\prime}+\Omega^{\prime}\right), \\
& L^{\prime}+\Omega^{\prime} \stackrel{\text { to }}{\longrightarrow} 180-I^{\prime}, \\
& \omega^{\prime}-\omega \stackrel{\text { to }}{\longrightarrow} 180-J, \\
& I^{\prime} \stackrel{\text { to }}{\longrightarrow} L+\Omega .
\end{aligned}
$$

From the spherical triangle ABC of Figure 1 we get

$$
\begin{aligned}
& \sin \left(\omega^{\prime}-\omega\right) \sin I^{\prime}=\sin (L+\Omega) \sin J, \\
& \sin (L+\Omega) \cos I=\cos \left(L^{\prime}+\Omega^{\prime}\right) \sin \left(\omega^{\prime}-\omega\right)+\sin \left(L^{\prime}+\Omega^{\prime}\right) \cos \left(\omega^{\prime}-\omega\right) \cos I^{\prime}, \\
& \cos (L+\Omega)=\cos \left(\omega^{\prime}-\omega\right) \cos \left(L^{\prime}+\Omega^{\prime}\right)-\sin \left(\omega^{\prime}-\omega\right) \sin \left(L^{\prime}+\Omega^{\prime}\right) \cos I^{\prime}, \\
& \sin \left(L^{\prime}+\Omega^{\prime}\right) \sin I^{\prime}=\sin I \sin (L+\Omega), \\
& \sin (L+\Omega) \cos J=\cos \left(\omega^{\prime}-\omega\right) \sin \left(L^{\prime}+\Omega^{\prime}\right)+\sin \left(\omega^{\prime}-\omega\right) \cos \left(L^{\prime}+\Omega^{\prime}\right) \cos I^{\prime} .
\end{aligned}
$$

Clearly the right hand side of each of the Equations (2) contained mixture of the unknown quantities (dented by primes) and known quantities (without primes) e.g. $\omega^{\prime}$ and $\omega$, while their left hand sides are known quantities. To overcome this difficulty, we have to apply the transformation rules of Equations (1) to Equations (2) and we get for the transformation from $I, \Omega, \omega$ to $I^{\prime}, \Omega^{\prime}, \omega^{\prime}$ the formulae:

$$
\begin{aligned}
& \sin \left(\omega^{\prime}-\omega\right) \sin I^{\prime}=\sin (L+\Omega) \sin J, \\
& \sin I^{\prime} \cos \left(L^{\prime}+\Omega^{\prime}\right)=\cos I \sin J+\sin I \cos J \cos (L+\Omega), \\
& \cos I^{\prime}=\cos J \cos I-\sin J \sin I \cos (L+\Omega), \\
& \sin \left(L^{\prime}+\Omega^{\prime}\right) \sin I^{\prime}=\sin I \sin (L+\Omega), \\
& \sin \left(\omega^{\prime}-\omega\right) \sin I^{\prime}=\cos J \sin I+\sin J \cos I \cos (L+\Omega) .
\end{aligned}
$$

2.2.2. The Basic Equations for the Transformation $I^{\prime}, \Omega^{\prime}, \omega^{\prime}$ to $I, \Omega, \omega$

By the same way as above, we get for the transformation from $I^{\prime}, \Omega^{\prime}, \omega^{\prime}$ to $I, \Omega, \omega$ the formulae: 


$$
\begin{aligned}
& \sin I \sin \left(\omega^{\prime}-\omega\right)=\sin \left(L^{\prime}+\Omega^{\prime}\right) \sin J, \\
& \sin I \cos \left(\omega^{\prime}-\omega\right)=\cos J \sin I^{\prime}-\sin J \cos I^{\prime} \cos \left(L^{\prime}+\Omega^{\prime}\right), \\
& \cos I=\cos I^{\prime} \cos J+\sin I^{\prime} \sin J \cos \left(L^{\prime}+\Omega^{\prime}\right), \\
& \sin I \sin (L+\Omega)=\sin I^{\prime} \sin \left(L^{\prime}+\Omega^{\prime}\right), \\
& \sin I \cos (L+\Omega)=-\cos I^{\prime} \sin J+\sin I^{\prime} \cos J \cos \left(L^{\prime}+\Omega^{\prime}\right) .
\end{aligned}
$$

\section{Unified Transformation Formula for J2000.0 and B1950.0 Keplerian Elements}

For practical applications, we can unified the two sets of Equations (1) and (2) as:

$$
\begin{aligned}
& S=\sin J=1.137895329 \times 10^{-4}, \\
& C=\cos J=0.999999993, \\
& X=S \sin \left(v+\Omega_{0}\right), \\
& Y=C \sin I_{0}+\eta S \cos I_{0} \cos \left(v+\Omega_{0}\right), \\
& Z=C \cos I_{0}-\eta S \sin I_{0} \cos \left(v+\Omega_{0}\right), \\
& Q=\sin I_{0} \sin \left(v+\Omega_{0}\right), \\
& T=S \eta \cos I_{0}+C \sin I_{0} \cos \left(v+\Omega_{0}\right) .
\end{aligned}
$$

where

- $I_{0}, \Omega_{0}, \omega_{0}$, the given elements.

- $\quad I^{\prime}, \Omega^{\prime}, \omega^{\prime}$, the required elements.

- $\eta$, an integer takes the values +1 or -1 such that:

- $\eta=+1$, for the transformation from the Keplerian orbital elements $\left(I_{0}, \Omega_{0}, \omega_{0}\right)$ with respect to reference system B1950.0 to the Keplerian orbital elements $\left(I_{1}, \Omega_{1}, \omega_{1}\right)$ with respect to reference system J2000.0.

- $\quad \eta=-1$, for the transformation from the Keplerian orbital elements $\left(I_{0}, \Omega_{0}, \omega_{0}\right)$ with respect to reference system J2000.0 to the Keplerian orbital elements $\left(I_{1}, \Omega_{1}, \omega_{1}\right)$ with respect to reference system B1950.0.

- $v=L$ if $\eta=1, v=L^{\prime}$ if $\eta=-1$ where $L \& L^{\prime}$, have the previous numerical values.

- The unified formulae for the required elements $I_{1}, \Omega_{1}, \omega_{1}$ in terms of the given elements $I_{0}, \Omega_{0}, \omega_{0}$ are:

$$
\begin{aligned}
& \omega_{1}=\omega_{0}+\tan ^{-1}(X / Y), \\
& I_{1}=\tan ^{-1}\left(\sqrt{X^{2}+Y^{2}} / Z\right), \\
& \Omega_{1}=-H+\tan ^{-1}(Q / T), \\
& H=\left\{\left(L+L^{\prime}\right)+\left(L^{\prime}-L\right) \eta\right\} / 2 .
\end{aligned}
$$

\section{Transformation Formulae between Equatorial and Ecliptic Orbital Elements}

Let the equatorial orbital elements of celestial body, be denoted by $\bar{\Omega}, \bar{i}, \bar{\omega}$ and its corresponding ecliptic elements be $\Omega, i, \omega$ (Figure 2 and Figure 3). The rest of the elements $(\tau, e, a)$, which determine the orbit of the body, do not change by changing the coordinates systems.

From the spherical triangle $\gamma \mathrm{NL}$ we get:

$$
\begin{aligned}
& \sin \bar{i} \sin \bar{\Omega}=\sin i \sin \Omega, \\
& \sin \bar{i} \cos \bar{\Omega}=\cos i \sin \varepsilon+\sin i \cos \varepsilon \cos \Omega, \\
& \cos \bar{i}=\cos i \cos \varepsilon-\sin i \sin \varepsilon \cos \Omega,
\end{aligned}
$$




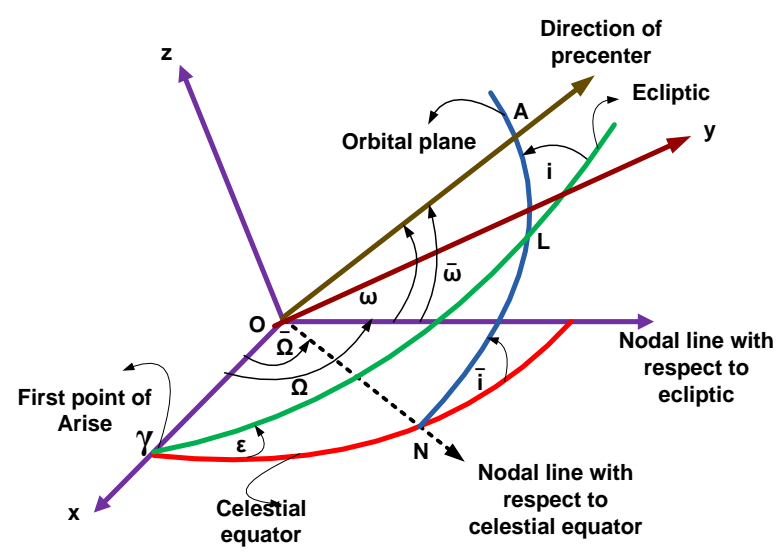

Figure 2. The orbit in space with respect to the fundamental planes.

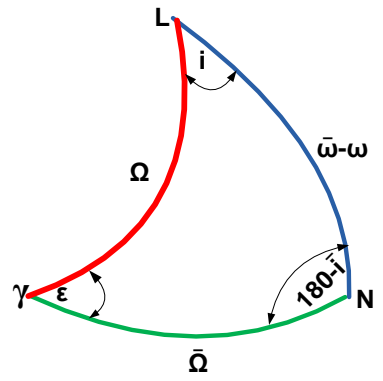

Figure 3. Equatorial and ecliptic orbital elements of celestial body.

$$
\begin{aligned}
& \sin \bar{i} \sin (\bar{\omega}-\omega)=\sin \varepsilon \sin \Omega, \\
& \sin \bar{i} \cos (\bar{\omega}-\omega)=\cos \varepsilon \sin i+\sin \varepsilon \cos i \cos \Omega .
\end{aligned}
$$

Equations (7) are to be used to obtain $\bar{i}, \bar{\Omega}, \bar{\omega}$ from $i, \Omega, \omega$.

Also

$$
\begin{aligned}
& \sin i \sin \Omega=\sin \bar{i} \sin \bar{\Omega}, \\
& \sin i \cos \Omega=-\cos \bar{i} \sin \varepsilon+\sin \bar{i} \cos \varepsilon \cos \bar{\Omega}, \\
& \cos i=\cos \bar{i} \cos \varepsilon+\sin \bar{i} \sin \varepsilon \cos \bar{\Omega}, \\
& \sin i \sin (\bar{\omega}-\omega)=\sin \bar{\Omega} \sin \varepsilon, \\
& \sin i \cos (\bar{\omega}-\omega)=\cos \varepsilon \sin \bar{i}-\sin \varepsilon \cos \bar{i} \cos \bar{\Omega} .
\end{aligned}
$$

\section{Unified Transformation Formulae for Equatorial and Ecliptic Orbital Elements}

For practical applications, we can unify the two sets of Equations (7) and (8) as:

$$
\begin{aligned}
& \sin i_{1} \sin \Omega_{1}=\sin i_{0} \sin \Omega_{0}=X, \\
& \sin i_{1} \cos \Omega_{1}=\sin i_{0} \cos \Omega_{0} \cos \varepsilon+\eta \cos i_{0} \sin \varepsilon=Y, \\
& \cos i_{1}=\cos i_{0} \cos \varepsilon-\eta \sin i_{0} \sin \varepsilon \cos \Omega_{0}=Z, \\
& \sin i_{1} \sin \eta\left(\omega_{1}-\omega_{0}\right)=\sin \varepsilon \sin \Omega_{0}=Q, \\
& \sin i_{1} \cos \eta\left(\omega_{1}-\omega_{0}\right)=\cos \varepsilon \sin i_{0}+\eta \sin \varepsilon \cos i_{0} \cos \Omega_{0}=T .
\end{aligned}
$$


where

- $i_{0}, \Omega_{0}, \omega_{0}$, the given elements.

- $i_{1}, \Omega_{1}, \omega_{1}$, the required elements.

- $\eta$, an integer takes the values +1 or -1 such that:

- $\eta=+1$, for the transformation from the ecliptic orbital elements $\left(i_{0}, \Omega_{0}, \omega_{0}\right)$ to equatorial orbital elements $\left(i_{1}, \Omega_{1}, \omega_{1}\right)$.

- $\eta=-1$, for the transformation from the equatorial orbital elements $\left(i_{0}, \Omega_{0}, \omega_{0}\right)$ to the ecliptic orbital elements $\left(i_{1}, \Omega_{1}, \omega_{1}\right)$.

Since the right hand side of each of the above equations is known (note $\varepsilon$ is known angle), consequently we can get $\left(i_{1}, \Omega_{1}, \omega_{1}\right)$ from

$$
\begin{aligned}
& i_{1}=\tan ^{-1}\left\{\sqrt{X^{2}+Y^{2}} / Z\right\}, \\
& \Omega_{1}=\tan ^{-1}\{X / Y\}, \\
& \omega_{1}=\omega_{0}+\eta \tan ^{-1}\{Q / T\} .
\end{aligned}
$$

\section{Computational Developments of J2000.0 and B1950.0 Keplerian Elements Transformations}

\subsection{Mathematica Module: KeplerB1950TJ2000}

\section{- Purpose}

Transfer the Keplerian orbital elements with respect to reference system B1950.0 to the Keplerian orbital elements with respect to reference system J2000.0 and vice versa.

- Input $i_{0}, \Omega_{0}, \omega_{0}$ (in radian), $\eta$.

- Output

- $i_{1}, \Omega_{1}, \omega_{1}$ (in radian).

- User defined procedures

Module: $\arctan$ (Appendix A).

Module: angle (Appendix A).

\section{- Module list}

$$
\begin{aligned}
&\text { KeplerB1950TJ2000[I0_, } \left.\Omega 0_{-}, \omega 0_{-}\right]:=\text {Module}\left[\left\{s=1.137895329 \times 10^{-4}, c=0.999999993, L\right.\right. \\
&=4.50001688 \times \mathrm{Pi} / 180, \mathrm{LD}=5.19856209 \times \mathrm{pi} / 180\}, \text { Which }[\eta>0, v=L, \eta \\
&<0, v=\mathrm{LD}] ; X=s \times \sin [v+\Omega 0] ; Y \\
&=c \times \sin [\mathrm{IO}]+\eta \times s \times \cos [\mathrm{I} 0] \times \cos [v+\Omega 0] ; Z \\
&=c \times \cos [\mathrm{I} 0]-\eta \times s \times \sin [\mathrm{I} 0] \times \cos [v+\Omega 0] ; Q=\sin [\mathrm{IO} 0] \times \sin [v+\Omega 0] ; T \\
&=\eta \times s \times \operatorname{Cos}[\mathrm{I} 0]+c \times \operatorname{Sin}[\mathrm{I} 0] \times \operatorname{Cos}[v+\Omega 0] ; \omega 1 \\
&=\operatorname{angle}[\omega 0+\eta \times \arctan [X, Y]] ; \mathrm{T} 1=\arctan \left[\operatorname{Sqrt}\left[X^{2}+Y^{2}\right], Z\right] ; H \\
&=((L+\mathrm{LD})+(\mathrm{LD}-L) \times \eta) / 2 ; \Omega 1=\operatorname{angle}[-H+\arctan [Q, T]] ;]
\end{aligned}
$$

\subsection{Numerical Examples}

Table 1 gives the transformations of the orbital Kepler elements 1950.0 to the corresponding elements J2000.0 and vice versa as computed from the above Module.

\section{Computational Developments of Equatorial and Ecliptic Orbital Elements Transformations}

\subsection{Mathematica Module: TranElements}

\section{- Module list}


TranElements[I0_, $\left.\Omega 0_{-}, \omega 0_{-}, \eta_{-}\right]:=$Module$[\{\varepsilon=23.4457889 \times \mathrm{Pi} / 180\}, X=\sin [\mathrm{I0}] \times \sin [\Omega 0] ; Y$

$$
\begin{aligned}
& =\sin [\mathrm{I} 0] \times \cos [\Omega 0] \times \cos [\varepsilon]+\eta \times \cos [\mathrm{I} 0] \times \sin [\varepsilon] ; Z \\
& =\cos [\mathrm{I} 0] \times \cos [\varepsilon]-\eta \times \sin [\mathrm{I} 0] \times \sin [\varepsilon] \times \cos [\Omega 0] ; Q=\sin [\Omega 0] \times \sin [\varepsilon] ; T \\
& =\cos [\varepsilon] \times \sin [\mathrm{I} 0]+\eta \times \sin [\varepsilon] \times \cos [\mathrm{I} 0] \times \cos [\Omega 0] ; \mathrm{I} 1 \\
& =\arctan \left[\operatorname{Sqrt}\left[X^{2}+Y^{2}\right],\right] ; \Omega 1=\arctan [X, Y] ; \omega 1 \\
& =\operatorname{angle}[\omega 0+\eta \times \arctan [Q, T]] ;]
\end{aligned}
$$

\subsection{Numerical Examples}

Table 2 gives the transformations of the ecliptic orbital elements to the equatorial orbital elements and vice versa.

Table 1. The transformations of the orbital Kepler elements 1950.0 to the corresponding elements J2000.0 and vice versa.

\begin{tabular}{ccccccc}
\hline No. & $I^{\circ}$ J2000.0 & $\Omega^{\circ}$ J2000.0 & $\omega^{\circ}$ J2000.0 & $I^{\circ}$ B1950.0 & $\Omega^{\circ}$ B1950.0 & $\omega^{\circ}$ B1950.0 \\
\hline 1 & 141.138 & 110.746 & 71.4059 & 141.135 & 110.054 & 71.4153 \\
2 & 100.297 & 185.304 & 238.78 & 100.290 & 184.606 & 238.779 \\
3 & 80.6362 & 231.315 & 114.576 & 80.6326 & 230.618 & 114.571 \\
4 & 112.57 & 53.6981 & 238.119 & 112.574 & 53.0019 & 238.125 \\
5 & 170.961 & 241.272 & 274.229 & 170.958 & 240.536 & 274.191 \\
6 & 139.921 & 179.841 & 171.182 & 139.915 & 179.142 & 171.182 \\
7 & 42.5016 & 186.461 & 244.876 & 42.4952 & 185.764 & 244.874 \\
8 & 96.6903 & 5.46674 & 252.29 & 96.6967 & 4.76833 & 252.291 \\
9 & 135.857 & 59.4065 & 121.267 & 135.86 & 58.7139 & 121.275 \\
10 & 86.4451 & 99.7851 & 160.631 & 86.4434 & 99.0862 & 160.637 \\
11 & 25.1243 & 299.08 & 227.031 & 25.1279 & 298.393 & 227.018 \\
12 & 99.5601 & 283.186 & 343.918 & 99.562 & 282.486 & 343.912 \\
13 & 101.079 & 281.101 & 306.966 & 101.08 & 280.401 & 306.959 \\
14 & 19.8767 & 29.158 & 308.421 & 19.8821 & 28.4495 & 308.431 \\
15 & 7.01984 & 144.568 & 74.358 & 7.01425 & 143.842 & 74.3855 \\
16 & 99.5299 & 336.251 & 107.795 & 99.5361 & 335.552 & 107.793 \\
17 & 166.572 & 177.496 & 257.746 & 166.565 & 176.796 & 257.745 \\
18 & 149.27 & 239.537 & 264.687 & 149.268 & 238.829 & 264.676 \\
19 & 48.5783 & 130.501 & 55.3847 & 48.5737 & 129.798 & 55.3908 \\
20 & 50.5978 & 2.61207 & 221.655 & 50.6043 & 1.91287 & 221.565 \\
\hline
\end{tabular}

Table 2. Transformations of the ecliptic orbital elements to the equatorial orbital elements and vice versa.

\begin{tabular}{ccccccc}
\hline No. & $i^{\circ}$ Ecliptic & $\Omega^{\circ}$ Ecliptic & $\omega^{\circ}$ Ecliptic & $i^{\circ}$ Equatorial & $\Omega^{\circ}$ Equatorial & $\omega^{\circ}$ Equatorial \\
\hline 1 & 7.60859 & 245.396 & 293.823 & 21.3627 & 340.702 & 197.087 \\
2 & 16.3726 & 238.797 & 186.535 & 20.2256 & 315.781 & 106.671 \\
3 & 65.1561 & 257.05 & 285.847 & 62.2011 & 268.744 & 259.849 \\
4 & 14.4351 & 171.36 & 182.167 & 9.41361 & 13.2365 & 340.731 \\
5 & 26.0028 & 24.8475 & 261.4 & 48.2195 & 14.3028 & 274.357 \\
6 & 32.4876 & 334.77 & 149.96 & 54.5113 & 343.669 & 137.938 \\
7 & 66.4539 & 188.537 & 66.7897 & 43.3465 & 191.435 & 61.8535 \\
8 & 158.761 & 143.292 & 346.835 & 137.694 & 161.233 & 7.52689 \\
9 & 54.892 & 179.516 & 114.373 & 31.4475 & 179.242 & 114.742 \\
10 & 92.4622 & 103.489 & 352.182 & 86.9441 & 103.369 & 14.9784 \\
11 & 156.25 & 250.335 & 104.782 & 141.796 & 217.824 & 67.4984 \\
12 & 55.6292 & 144.095 & 322.863 & 38.3768 & 128.768 & 344.94 \\
13 & 46.5676 & 26.1908 & 352.194 & 68.2823 & 20.2131 & 3.08979 \\
14 & 31.078 & 297.183 & 169.174 & 46.2172 & 320.504 & 139.818 \\
15 & 99.8029 & 85.0414 & 331.311 & 100.958 & 89.3931 & 355.124 \\
\hline
\end{tabular}


In concluding, the present paper introduced two unified and simple algorithms that are capable of executing calculations in both directions and in one program run to the 1) transformations between J2000.0 Keplerian orbital elements and B1950.0 elements and 2) transformations between the equatorial orbital elements and the ecliptic orbital elements. The algorithms are elaborated using Mathematica package, which is qualified for accurate computations. The proposed algorithms are checked by numerical examples given in Table 1 and Table 2 .

\section{References}

[1] Capitaine, N. and McCarthy, D. (2004) American Astronomical Society Meeting 204, \#28.01. Bulletin of the American Astronomical Society 36, p.694.

[2] Standish, E.M. (1982) Conversion Positions and Proper Motions from B1950.0 to the IAU System at J2000.0. Astronomy and Astrophysics, 115, 20-22.

[3] Murray, C.A. (1989) The Transformation of Coordinates between the Systems of B1950.0 and J 2000.0, and the Principal Galactic Axes Referred to J2000.0. Astronomy and Astrophysics, 218, 325-329.

[4] Aoki, S., Soma, M., Kinoshita, H. and Inoue, K. (1983), Conversion Matrix of Epoch B1950.0 FK 4-Based Positions of Stars to Epoch J2000.0 Positions in Accordance with the New IAU Resolution. Astronomy and Astrophysics, 128, 263-267.

[5] Todhunter, M.A. (1925) Spherical Trigonometry. Macmillan \& Co., London.

[6] Seidelmann, P.K. (2005) Explanatory Supplement to the Astronomical Almanac. University of Science Books, Sausalito. 


\section{Appendix A: The User Defined Procedures}

A-1 User defined procedure: angle

Mathematica Module: angle

- Purpose

To reduce the angle $x$ in radian to the interval $[0,2 \pi]$

- Input

The value of $x$

- Output

The angle $x \in[0,2 \pi]$

- User defined procedures

None

- Module list

angle $x_{-}$: Module, Mod $x, 2$

- Notes

1. The above procedure is valid for positive as well as for negative angles.

2. For all values $x= \pm 2 n x$ where $n$ is positive integer number, the reduced angle is equal to zero.

3. The given angle $x$ and the reduced angle are the same if $x \in[0,2 \pi]$.

A-2 User defined procedure: Arctan $(y / x)$

When the inverse function of "tan" is taken an ambiguity arises which has to be cleared up. The angle $\tan ^{-1}(y / x)$ lies in the first quadrant if $x>0, y>0$, in the second quadrant if $x<0, y>0$, in the third quadrant if $x>0, y<0$, finally in the fourth quadrant if $x<0, y<0$. To obtain the correct quadrant, add or subtract $2 \pi$ or $\pi$ from the angle $\tan ^{-1}(y / x)$.

Mathematica Module: arctan

- Purpose

To find the correct quadrant of the angle $\tan ^{-1}(y / x)$ in radian.

- Input

$Y, x$

- Output

The angle $\tan ^{-1}(y / x)$ in radian.

- User defined procedures

None

- Module list

$\arctan \left[y_{-}, x_{-}\right]:=\operatorname{Module}[\{p, z\}, p=N[\pi] ; \operatorname{Which}[x !=0, z=\operatorname{ArcTan}[\operatorname{Abs}[y / x]] / / N$,

$x=0, z=p l 2]$; Which $[x>=0 \& \& y>=0, z \times \operatorname{Sign}[y], x<0 \& \& y>=0,-z \times p$,

$x<0 \& \& y<0, z+p, x>=0 \& \& y<0,-z+2 \times p]]$;

angle $\left[x_{-}\right]:=\operatorname{Module}[\{\}, \operatorname{Mod}[x, 2 \times \pi]]$;

- Notes

The usage of the Mathematica built in $\operatorname{ArcTan}[\mathbf{y} / \mathbf{x}]$ and the function $\arctan [y, x]$ of the above procedure for calculating the angle $\tan ^{-1}(y / x)$ are illustrated as follows.

- If $L=y / x$ is negative, the computer cannot determine the source of this negative sign, is it from the denominator or from the numerator, so it evaluates ArcTan[|L|] an then multiply the result by (-1). For examples:

- $\operatorname{ArcTan}[4 /-10]=-\operatorname{ArcTan}[4 / 10]=-0.3805^{r}=-21.801^{\circ}$, while $\arctan [4,-10]$ of the above procedure gives $2.7611^{r}=158.199^{\circ}$.

- $\operatorname{ArcTan}[-17 / 4]=-\operatorname{ArcTan}[17 / 4]=-1.3397^{r}=-76.759^{\circ}$, while $\arctan [-17,4]$ of the above procedure gives $4.9435^{r}=283.242^{\circ}$.

- If $L=y / x$ is positive, the computer cannot determine the source of this positive sign, is it because both denominator and numerator are positive, or both are negative, so it evaluates ArcTan[L]. For example:

- $\operatorname{ArcTan}[-15 /-6]=\operatorname{ArcTan}[15 / 6]=1.1903^{r}=68.198^{\circ}$, while $\arctan [-15,-6]$ of the above procedure gives $4.3319^{r}=248.199^{\circ}$

The only case in which $\operatorname{ArcTan}[\mathbf{y} / \mathbf{x}]=\arctan [y, x]$ is when $x>0, y>0$. 
Scientific Research Publishing (SCIRP) is one of the largest Open Access journal publishers. It is currently publishing more than 200 open access, online, peer-reviewed journals covering a wide range of academic disciplines. SCIRP serves the worldwide academic communities and contributes to the progress and application of science with its publication.

Other selected journals from SCIRP are listed as below. Submit your manuscript to us via either submit@scirp.org or Online Submission Portal.
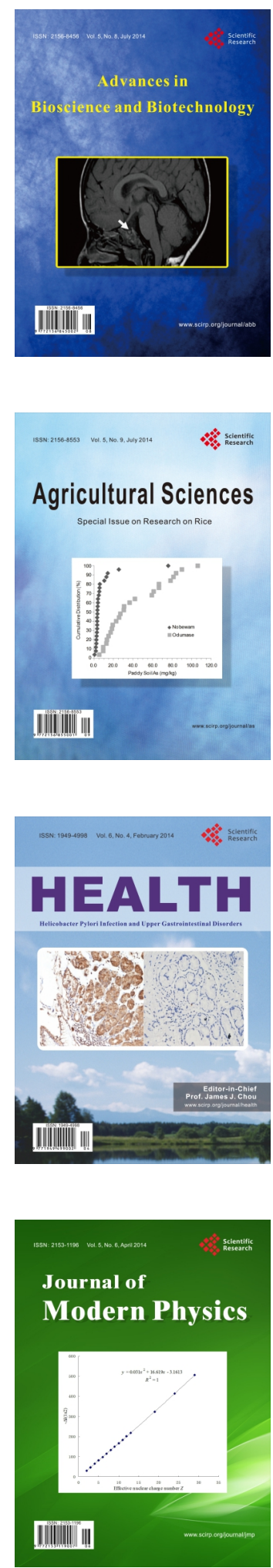
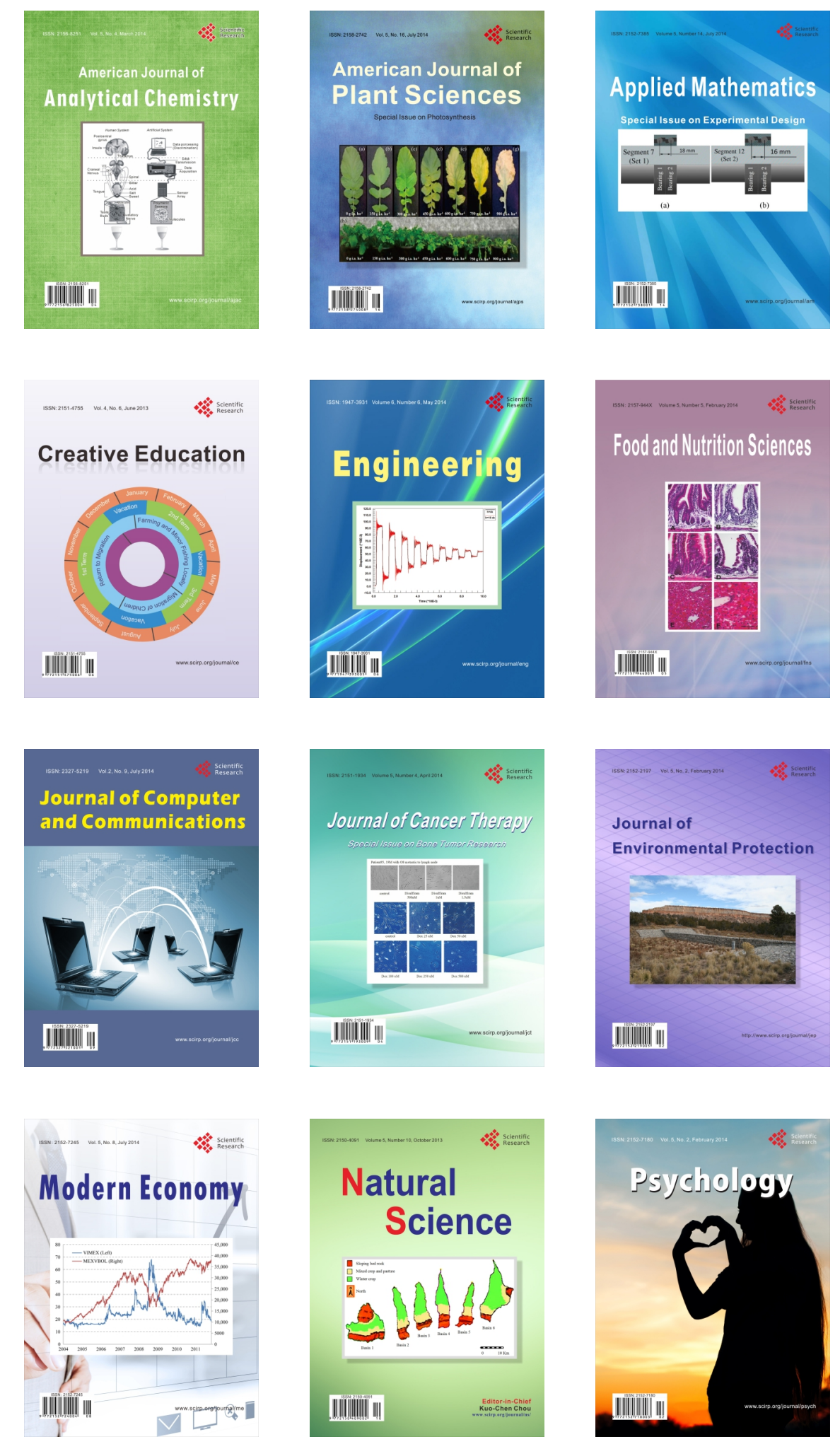